\title{
The story of the lost twins: decoding the genetic identities of the Kumhar and Kurcha populations from the Indian subcontinent
}

\author{
Ranajit Das ${ }^{1^{*}}$ D, Vladimir A. Ivanisenko ${ }^{2,3}$, Anastasia A. Anashkina ${ }^{4,5}$ and Priyanka Upadhyai ${ }^{6}$
}

From 11th International Young Scientists School "Systems Biology and Bioinformatics" - SBB-2019

Novosibirsk, Russia. 24-28 June 2019

\begin{abstract}
Background: The population structure of the Indian subcontinent is a tapestry of extraordinary diversity characterized by the amalgamation of autochthonous and immigrant ancestries and rigid enforcement of sociocultural stratification. Here we investigated the genetic origin and population history of the Kumhars, a group of people who inhabit large parts of northern India. We compared 27 previously published Kumhar SNP genotype data sampled from Uttar Pradesh in north India to various modern day and ancient populations.

Results: Various approaches such as Principal Component Analysis (PCA), Admixture, TreeMix concurred that Kumhars have high ASI ancestry, minimal Steppe component and high genomic proximity to the Kurchas, a small and relatively little-known population found $\sim 2500 \mathrm{~km}$ away in Kerala, south India. Given the same, biogeographical mapping using Geographic Population Structure (GPS) assigned most Kumhar samples in areas neighboring to those where Kurchas are found in south India.

Conclusions: We hypothesize that the significant genomic similarity between two apparently distinct modern-day Indian populations that inhabit well separated geographical areas with no known overlapping history or links, likely alludes to their common origin during or post the decline of the Indus Valley Civilization (estimated by ALDER). Thereafter, while they dispersed towards opposite ends of the Indian subcontinent, their genomic integrity and likeness remained preserved due to endogamous social practices. Our findings illuminate the genomic history of two Indian populations, allowing a glimpse into one or few of numerous of human migrations that likely occurred across the Indian subcontinent and contributed to shape its varied and vibrant evolutionary past.
\end{abstract}

Keywords: Kumhar, Kurchas, Indus Valley civilization, South Asian population history

\footnotetext{
* Correspondence: das.ranajit@gmail.com

${ }^{1}$ Yenepoya Research Centre (YRC), Yenepoya (Deemed to be University), Mangalore, Karnataka, India

Full list of author information is available at the end of the article
}

(c) The Author(s). 2020 Open Access This article is licensed under a Creative Commons Attribution 4.0 International License, which permits use, sharing, adaptation, distribution and reproduction in any medium or format, as long as you give appropriate credit to the original author(s) and the source, provide a link to the Creative Commons licence, and indicate if changes were made. The images or other third party material in this article are included in the article's Creative Commons licence, unless indicated otherwise in a credit line to the material. If material is not included in the article's Creative Commons licence and your intended use is not permitted by statutory regulation or exceeds the permitted use, you will need to obtain permission directly from the copyright holder. To view a copy of this licence, visit http://creativecommons.org/licenses/by/4.0/ The Creative Commons Public Domain Dedication waiver (http://creativecommons.org/publicdomain/zero/1.0/) applies to the data made available in this article, unless otherwise stated in a credit line to the data. 


\section{Background}

The Indian subcontinent and adjoining regions in South Asia have been a cradle for several waves of human migration during Paleolithic, Neolithic Periods, Bronze and Iron Age [1-6]. The genetic and ethnolinguistic landscape of the Indian subcontinent is remarkably heterogeneous and sculpted by the confluence of the indigenous people with immigrants that arrived into India following diverse routes [7-13]. The extant Indian gene pool is composed of largely four ancestral genetic components, namely Ancestral North Indian (ANI), Ancestral South Indian (ASI), Ancestral Tibeto-Burman (ATB), and Ancestral Austro-Asiatic (AAA) [14-16]. Recent studies dissecting the complex genetic history of South Asia suggested that a South Asia Hunter Gatherer lineage with close proximity to the present day Andamanese (AASI) admixed with individuals related to Iranian agriculturalists from Zagros mountains, Iran and West_Siberian_HG (West Siberian Hunter Gatherers) forming the Indus_Periphery gene pool, in the larger Indus valley area during the 3rd millennium BCE, and may be a vital ancestral source for the subsequent peopling of South Asia [17]. Consistent with previous evidences [11, 18] the autochthonous Indian ancestral lineages prior to their admixture with West Eurasians, likely split during eastward migration of the anatomically modern humans, out of Africa, later giving rise to AASI groups [17]. The ANI and ASI gene pools arose subsequently around the 2nd millennium BCE, concurrent with the decline of the Indus Valley civilization (IVC) [19] that propelled a massive upheaval in human settlements across northern parts of the Indian subcontinent. The southward dispersal of Steppe_MLBA (later Middle to late Bronze Age Steppe) populations occurred around this time into South Asia [20-22]; it is envisioned that the Indus_Periphery related groups admixed with the Steppe_MLBA immigrants to form the ANI, while additional Indus Periphery people migrated further south and eastward within peninsular India to mingle with AASI and formed the ASI [17]. The distinctive population structure of the Indian subcontinent is a unique amalgamation resulting from the extensive and intricate percolation of people across it for long periods together with the rigorous enforcement of sociocultural practices, such as endogamy in many groups. Interrogation of population structure, relatedness and ancestry of Indian populations provide valuable insight to not only reconstruct their evolutionary past but may also have important implications in medical genetics and understanding relevant disease biology.

Here we have investigated the population history of the Kumhars, a north Indian population that has likely been practicing endogamy over long periods of time, as evidenced by their Identical by descent (IBD) scores that are significantly higher than that of the Ashkenazi Jews and the Finns [23]. Kumhars are found throughout large parts of northern, western, and eastern India, as well as in Pakistan. The name 'Kumhar' is derived from the Sanskrit term 'Kumbhakar', which literally means earthenpot makers alluding to their ancestral way of earning a living [24]. Interestingly, the potters from Amritsar, Punjab in north India are also known as Kulal or Kalal, a term phonetically similar to Kulala, a group of people from Kasaragod district of the southern Indian state of Kerala, whose traditional occupation is also pottery. The phonetic similarity between the two terms is potentially due to their common origin from the Yajurveda, an ancient Vedic Sanskrit text where potters were termed as 'Kulals' [25]. In this study we aimed to delineate the population history of Kumhars and examined their genomic similarity with other populations from the Indian subcontinent. To this end we assessed 27 previously published Kumhar samples, which were sampled from the north Indian state of Uttar Pradesh and compared to 2013 modern day South Asian populations [16, 23].

\section{Results}

Clustering of Kumhars in the context of other south Asian populations

Principal component analysis (PCA) of South Asian samples exhibited previously described $[14,26]$ ANI ASI -AAA cline along the horizontal principal component (PC1) with Ashkenazi Jews, Kalash and other Pakistani populations, and Shia Iranians from Hyderabad clustering at one extreme of the cline, and Juang and other AAA populations congregating at the other extreme (Fig. 1 and Additional file 1 Fig. S2). Concurrent with our previous study [26], ASI-AAA-Ancestral Tibeto-Burman (ATB) contrast was observed along the vertical principal component (PC2) with Juangs clustering at one end, while Nyshi and other ATB populations clustered at the other end. Out of 27 Kumhar samples employed in this study, barring three (stockplate_14_C2, stockplate_14_C4, stockplate_14_C6), the remaining clustered with ASI and AAA samples, largely overlapping with tribal populations from Kerala such as the Kurchas. According to PCA, the only North Indian population that revealed genomic proximity to Kumhars, were the Syons from Uttarakhand. Further we note that the three above-mentioned outlier Kumhar samples overlapped with a population cluster that largely comprised of various non-Brahmin backward castes from Uttar Pradesh.

Weighted pairwise $F_{\mathrm{ST}}$ between Kumhars and 63 selected populations across India using Weir and Cockerham approach [27] implemented in PLINK v1.9 revealed the Kumhars to be genetically almost identical to Kurchas from Kerala in southern India (weighted $F_{\mathrm{ST}}=$ 

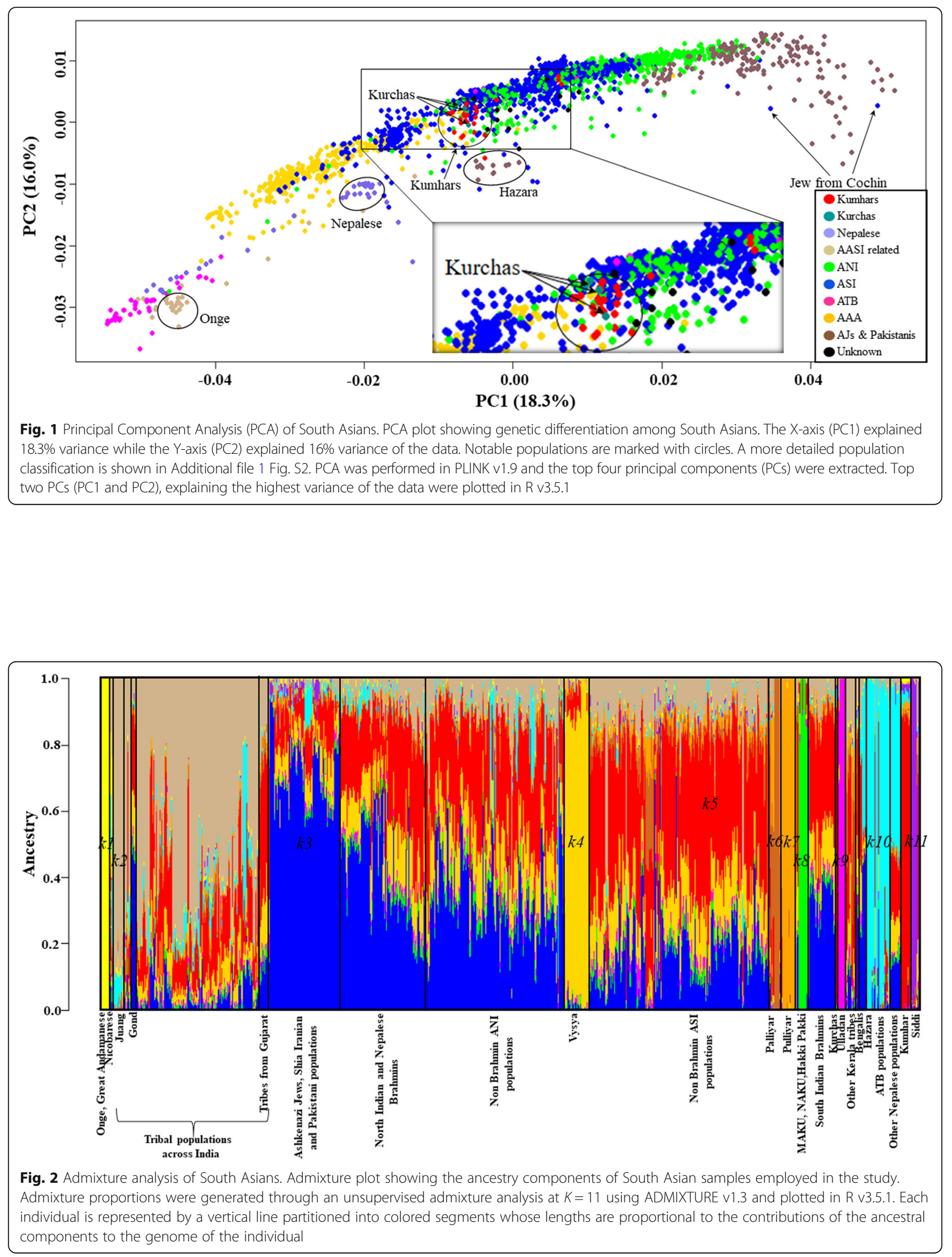
0.0008) (Additional file 1 Table S1). Among the remaining populations, Kumhars were genetically closest to Kurumbas from Kerala, south India (weighted $F_{\mathrm{ST}}=$ 0.019) followed by Vishwabrahmins from Andhra Pradesh, south India (weighted $F_{\mathrm{ST}}=0.0192$ ) and Chakkiliyans from Tamil Nadu, south India (weighted $F_{\mathrm{ST}}=$ 0.0196), and farthest from largely homogeneous populations from Kerala and Tamil Nadu such as Adiyan (weighted $F_{\mathrm{ST}}=0.044$ ), Paniyas (weighted $F_{\mathrm{ST}}=0.054$ ), Narikuruvar (weighted $F_{\mathrm{ST}}=0.055$ ), Pulliyar (weighted $F_{\mathrm{ST}}=0.056$ ), Malaikuravar (weighted $F_{\mathrm{ST}}=0.07$ ) and Ulladan (weighted $F_{\mathrm{ST}}=0.077$ ). Weighted pairwise $F_{\mathrm{ST}}$ between Kurchas and the same 63 populations across India revealed similar results, indicating that Kurchas are genetically more similar to Kumhars than any other Indian populations including its neighboring ones (Additional file 1 Table S2).

The genomic ancestry of all 2040 individuals present in the Modern South Asian only dataset was estimated using the model-based clustering algorithm ADMIXTURE v1.3 [28]. The lowest CVE was estimated for $K=$ 11 (Additional file 1 Fig. S1). At $K=11$, discernible degree of genetic admixture was observed between ANI and ASI populations (Fig. 2). Onge ( $k 1$, yellow), Juang (k2, tan), Ashkenazi Jews and Shia Iranians (k3, blue), Vysya (k4, gold), Kumhar and Kurchas (k5, red), Palliyar (k6, chocolate brown), Pulliyar ( $k 7$, orange), Malaikuravar, Narikuruvar and Hakki Pakki (k8, green), Ulladan (k9, magenta), ATB (k10, cyan), and Siddi (k11, purple) populations were assigned to distinct clusters. Congruent with previous studies $[14,16,26]$, Fig. 2 revealed that most South Asians have variable fractions of blue ( $k 3$, likely derived from Bronze Age Steppe populations), red ( $k 5$, likely derived from ASI populations) and tan $(k 2$, likely derived from Ancient Ancestral South Indians: AASI populations). Component $k 5$, which was assigned to Kumhars and Kurchas, was found to be present in discernibly higher proportion among most non-Brahmin south Indian populations, indicating genomic similarity between Kumhars and ASI populations potentially linked to their common origin and admixture history. Further, the Bronze Age Steppe ancestry proportion was found to be the lowest in Kumhars compared to other populations from Uttar Pradesh and Uttarakhand (Fig. 3), indicating their distinct origin. We note that most Kumhar samples were found to have $<1 \%$ Bronze Age Steppe ancestry, except stockplate_14_C2, stockplate_14_C4, stockplate_14_C6 (all three have 25\% Steppe related ancestry), and were also found to be outliers in principal component analysis. Both PCA and ADMIXTURE analysis suggested that origin of these three Kumhar samples was divergent from the rest.

We employed TreeMix v.1.12 [29] to investigate the pattern of population splits and mixtures among selected South Asian populations. Similar to PCA, $F_{\mathrm{ST}}$ and ADMIXTURE analyses, the ML tree generated by TreeMix revealed high degree of genetic relatedness between Kumhars and Kurchas, with Kurumba (Kerala) and Sugali (Andhra Pradesh) populations as their sister groups (Fig. 4). Overall, all clustering approaches employed in this study revealed high proximity between Kumhar and Kurchas samples, and high degree of genetic similarity

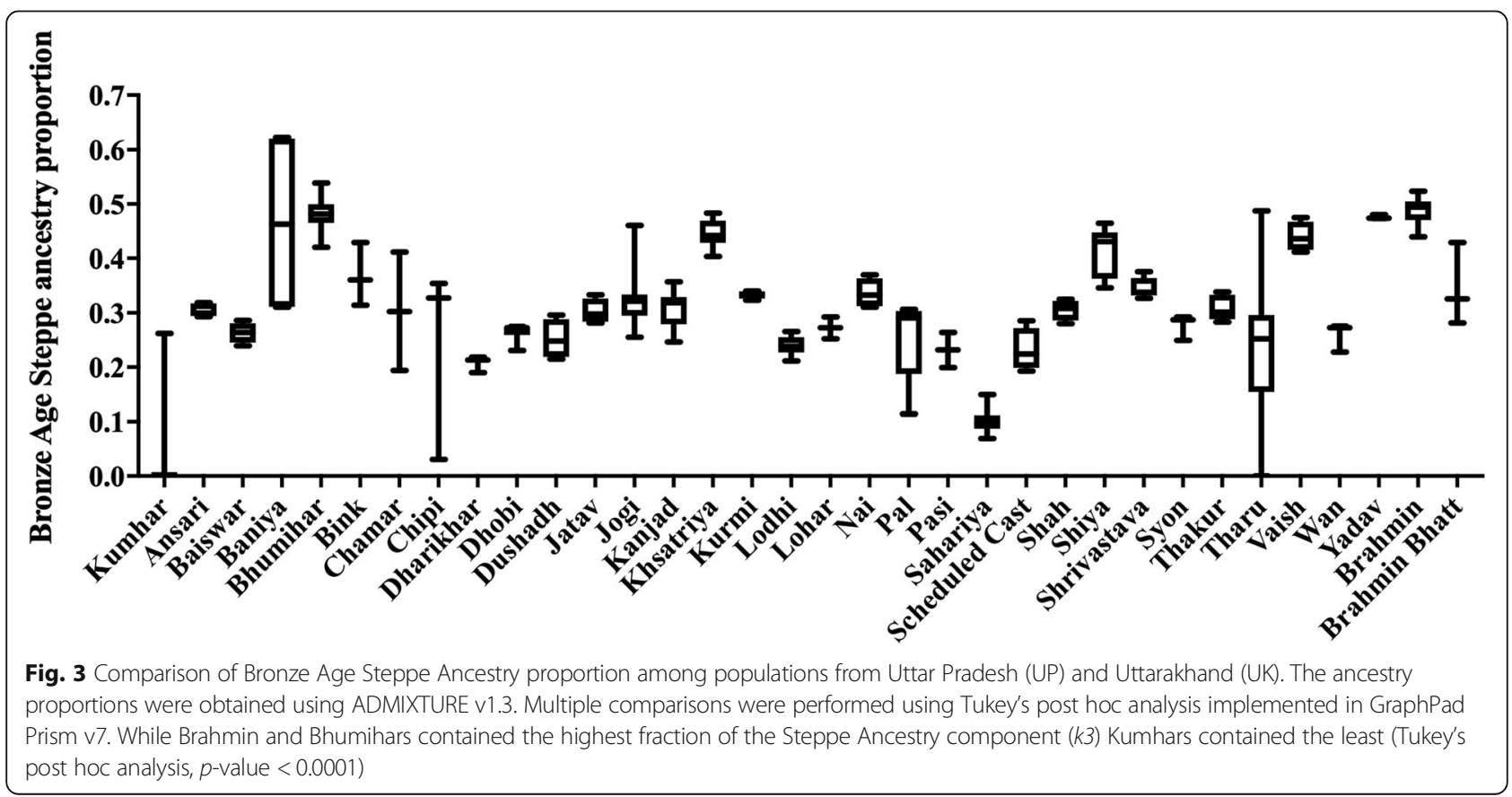




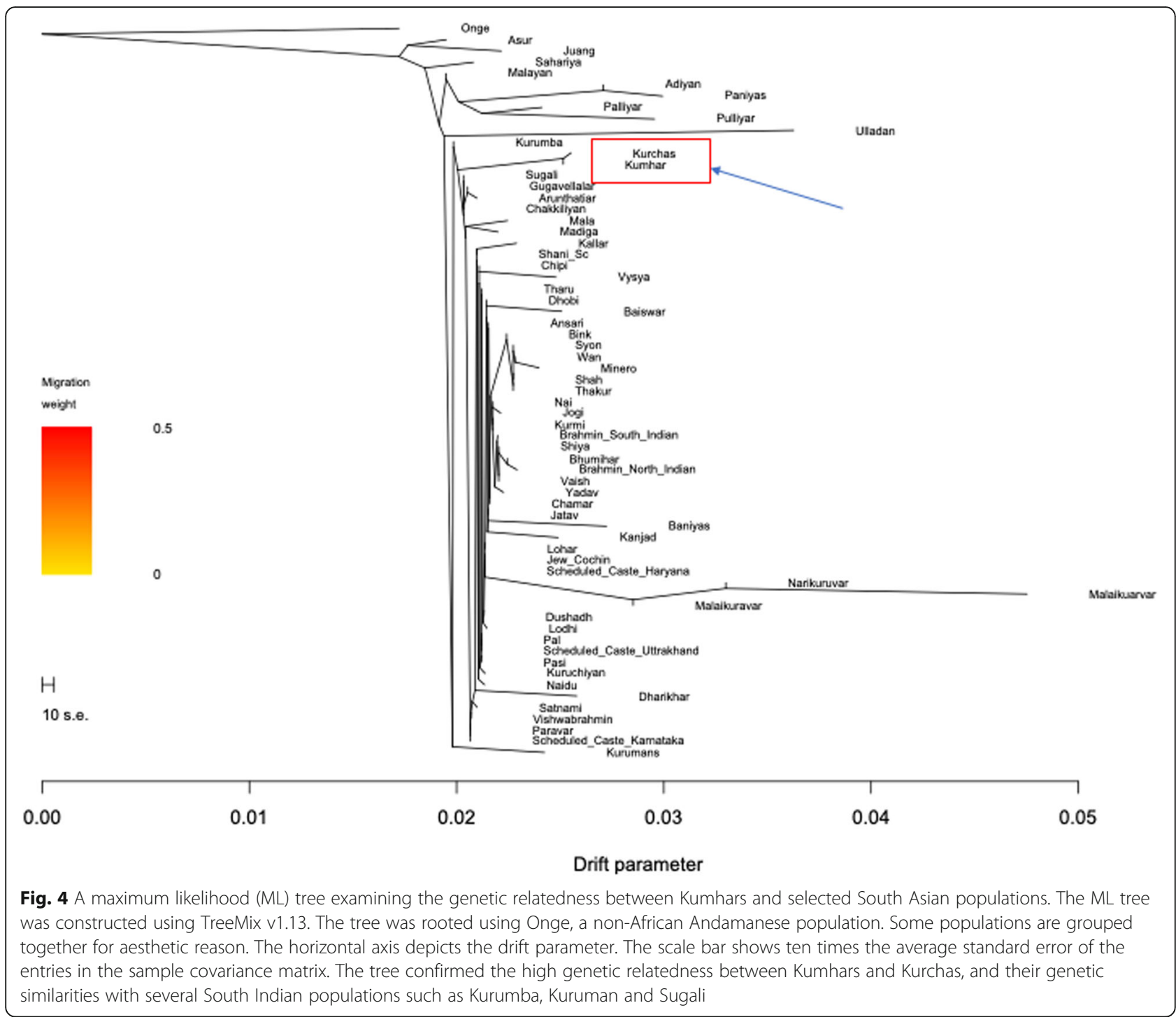

between Kumhars and several modern-day South Indian populations. On the contrary, barring the three outlier Kumhar samples, the remainder had very little genomic proximity towards other populations from the same geographic location in Uttar Pradesh and Uttarakhand.

We applied ALDER v.1.02 [30] to investigate the approximate time of admixture between Kumhars and other South Asians. 39\% of the successful results in terms of LD decay included Dravidian speaking Brahui population from Pakistan, alongside an Austroasiatic speaking (Oraon, Ho) or an AASI related (Juang) population or a backward caste population (Mohali, Kandha, Gond and Khairwar) from north and central India (Additional file 1 Table S3). Our results indicate that the admixture event that potentially gave rise to Kumhars likely occurred $130-200$ generations or $3640-5600$ years ago, assuming a generation time of 28 years. This timeline (3600-1640 BCE) likely coincides with or after the decline of the IVC $[19,31]$.

\section{Biogeographical mapping of Kumhar samples employed in the study}

Biogeographical mapping of Kumhar samples was performed using the GPS algorithm. Barring stockplate_14 C2, stockplate_14_C4, stockplate_14_C6, and CCMB_ PL_9_298, GPS assigned all 23 Kumhar samples to south-western Karnataka across Western Ghats in southern India with 16 being localized at the KarnatakaKerala border (Fig. 5). Stockplate_14_C6 was positioned $\sim 80 \mathrm{~km}$ southeast of Hubballi, northern Karnataka. Stockplate_14_C4 was localized $\sim 130 \mathrm{~km}$ east of Madurai, along coastal Tamil Nadu, in south India. $C C M B_{-}$ PL_9_298 was assigned $<50 \mathrm{~km}$ west of Visakhapatnam, Andhra Pradesh, in south India. Only one Kumhar 


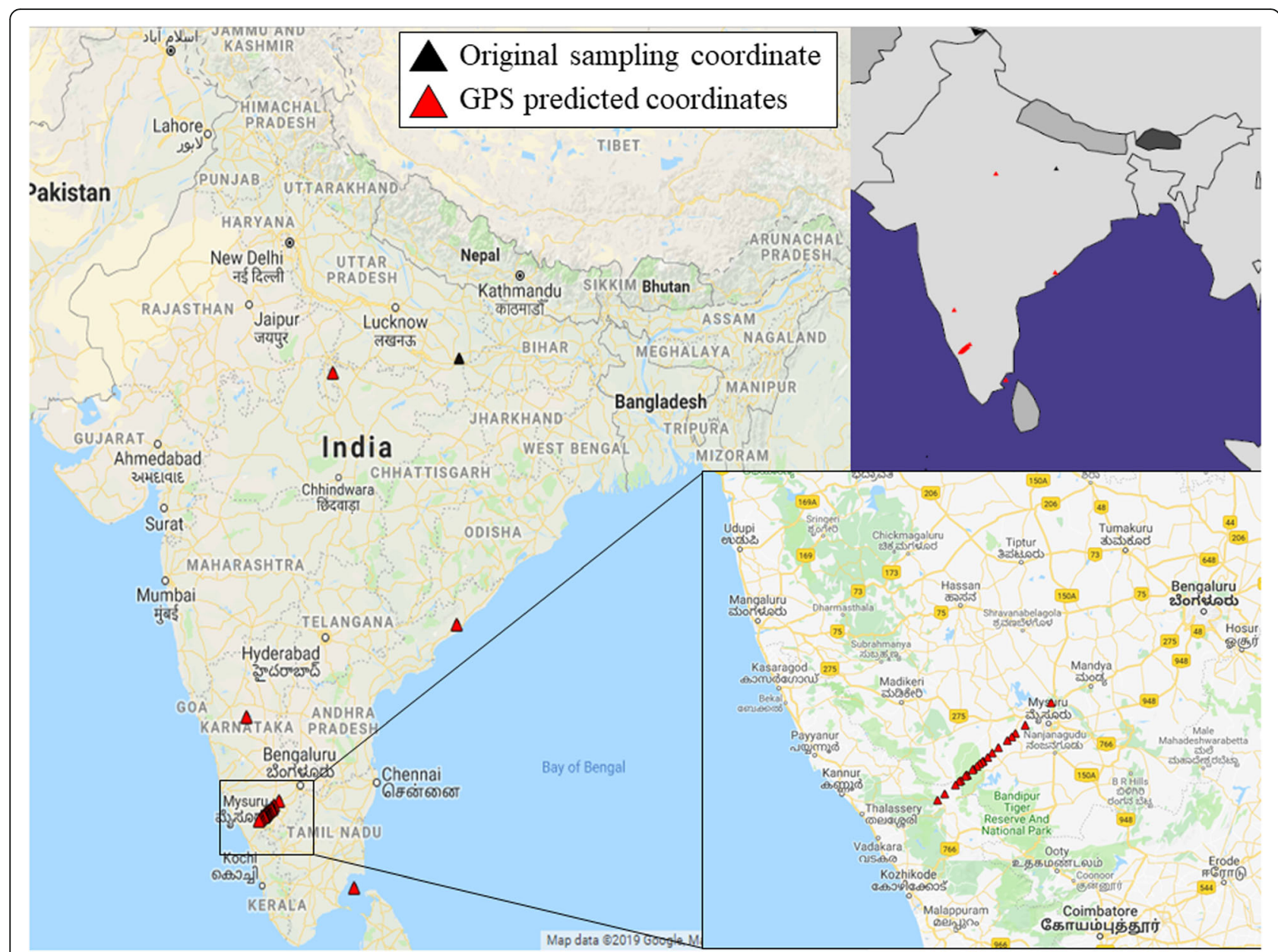

Fig. 5 Maps depicting the GPS predicted locations of the Kumhar samples. GPS predicted coordinates were plotted in Google Maps (Google LLC) as well as using 'rworldmap' package implemented in R v3.5.1. The black triangle represents the original sampling location of Kumhars and the red triangles represent GPS predicted locations of Kumhars. To note, GPS localized 23 out of 27 Kumhar samples to south-western Karnataka across Western Ghats in southern India with 16 being localized at the Karnataka-Kerala border

sample, stockplate_14_C2 was assigned by GPS to Uttar Pradesh in north India, from where all Kumhar samples had been actually collected [23]; it was localized $~ 300$ $\mathrm{km}$ southwest of Kanpur and $\sim 70 \mathrm{~km}$ south of Jhansi. The assignment of 26 out of 27 Kumhars sampled from Uttar Pradesh, north India to South India further confirms their high genomic proximity with populations of ASI ancestry and genetic distinctness from other populations found in overlapping and neighboring areas of their geographic sampling source.

\section{Determination of ancestry proportions in Kumhars in a global context}

We used qpAdm [32] implemented in AdmixTools v5.1 [33] to estimate ancestry proportions in the South Asians. The Ancient-Modern dataset comprising of 4575 ancient and modern-day individuals worldwide was employed for this analysis. All South Asians were modelled as a combination of three source populations namely Andaman Islanders (Onge), Steppe-related (Steppe-MLBA) and Iran-Turan-related (Indus_Periphery) as Left (Test, Onge, Steppe-MLBA, Indus_Periphery) and $\mathrm{O} 8$ was used as the 'Right' outgroup (see Methods). Congruent with ADMIXTURE analysis, qpAdm analysis revealed that Kumhars (10.5\%) are among the three populations from Uttar Pradesh, north India with low Bronze Age Steppe ancestry (Additional file 1 Table S4); the other two populations being Dharikhar $(10.2 \%)$ and Sahariya tribes $(0 \%)$. When we repeated the $q p A d m$ analysis for Kumhars excluding the outlier Kumhars (stockplate_14_C2, stockplate_14_C4 and stockplate_14_C6), the Steppe ancestry reduced to $9.5 \%$ and Kumhars emerged as the population with the second lowest Steppe ancestry after Sahariyas. It is noteworthy that Onge, Steppe_MLBA and Indus_Periphery ancestral component in Kumhars, after removal of the three outliers (49.2, 9.5 and $41.2 \%$ respectively), was found to be very similar to that in Kurchas (51.4, 9.3 and 39.3\% 
respectively) and Kurumans from Kerala (46.2, 9.3 and 44.5\% respectively), and Chakkiliyans from Tamil Nadu (47.3, 9.4 and $43.3 \%$ respectively), in south Indian reflecting the significant genomic proximity between Kumhars and ASI populations.

\section{The direction of gene flow: genetic similarities between Kumhars and Kurchas}

We found positive Z-scores for all combinations of South Asian populations (X) employed in this study, indicating gene flow between Kumhars (W) and Kurchas (Y) (Additional file 1 Table S5). The positive Z-scores obtained from D-statistical analysis indicates that Kumhars are genetically closer to Kurchas than to any other Indian group employed in this study.

\section{Discussion}

Populations from the Indian subcontinent are envisaged as a Pleistocene gene pool [10,34-36] and are a mélange of varied indigenous and immigrant ancestries, which together with the extraordinary diversity in geographical niches and sociocultural stratification has resulted in its complex genetic history. Here we investigated the genetic origin and population history of the Kumhars, a group of people who traditionally worked as potters and are found over large parts of north, west and east India.

Pottery is the art of creating objects from non-metallic minerals, such as earthenware, porcelain by molding them when wet and subsequently firing them at high temperatures. It was practiced by potters referred to as Kumhars in northern, western and eastern regions of the Indian subcontinent. The origin of pottery in the Indian subcontinent can be traced back to cord-impressed style of ceramic ware from the Mesolithic period, found at the site of Lahuradewa, dating back to 7000-6000 BCE [37]. Evidences of both handmade and wheel-made forms of pottery dating back to the IVC have been obtained. The Jhukar phase of pottery corresponding to the Jhukar archaeological type-site in Sindh was coincident with urbanization in the late Harappan period [38]. This was followed by the crude handmade pottery of the Jhangar phase [38] likely reflecting a largely nomadic and pastoralist population of West Asian immigrants. The decline of the IVC and the subsequent peopling of the vast Gangetic plains in central Indian subcontinent was marked by handmade and unpainted pottery forms, such as those of the Swat grave culture and ochre colored pottery culture that further likely coincided with West Eurasian migration into the Indian subcontinent. This was followed by black and red ware and subsequently the painted grey ware cultures of pottery that likely concurred with south and eastward migration of people in the peninsular India and the formation of the ASI [39].
In India, Kumhars and their Southern counterparts such as Kulals (in Kerala), Kummara (Andhra Pradesh and Telangana), Kumbara and Kummari (Andhra Pradesh) are synonymous with pottery. We interrogated 27 previously published Kumhar SNP genotype data [23] and compared them to various modern day and ancient populations.

PCA revealed that except three (stockplate_14_C2, stockplate_14_C4, stockplate_14_C6), all Kumhars congregated with those of ASI and AAA ancestries, largely overlapping with tribal populations from Kerala, in southern India, such as the Kurchas (Fig. 1). Similarly weighted pairwise $F_{\mathrm{ST}}$ using the Weir and Cockerham approach suggested that the Kumhars were genetically almost identical to Kurchas from Kerala, south India (weighted $F_{\mathrm{ST}}=0.0008$ ), followed by Kurumbas from Kerala (weighted $F_{\mathrm{ST}}=0.019$ ), Vishwabrahmins from Andhra Pradesh, south India (weighted $F_{\mathrm{ST}}=0.0192$ ) and Chakkiliyans from Tamil Nadu, south India (weighted $F_{\mathrm{ST}}=0.0196$ ) (Additional file 1 Table S1). The strong genomic proximity of Kumhars with Kurchas was further corroborated by TreeMix analysis (Fig. 4). Consistent with this, Admixture analysis also reflected a predominant ASI ancestry among the Kumhars that is also shared by Kurchas and other non-Brahmin south Indian populations (Fig. 2). For most Kumhar samples PCA, Admixture and $q p A d m$ concurred on the presence of a minimal Steppe ancestral component (Fig. 3 and Additional file 1 Table S3).

Given the high genetic similarity between Kumhars and Kurchas it is unsurprising that biogeographical mapping of the Kumhars assigned all but one sample to southern India (Fig. 5). Notably 23 Kumhar samples were positioned to south-western Karnataka across Western Ghats in southern India with 16 being localized at the borders of the Indian states of Karnataka and Kerala, and adjoining the geographic region of Wayanad, which is the native abode of the Kurcha population.

Finally using ALDER we estimated that the Kumhar gene pool likely arose 130-200 generations or 36405600 years ago coinciding with two important events that potentially occurred during and/or after the decline of IVC [19, 31]: (a) the emergence of the ASI group, which began $\sim 3000 \mathrm{BCE}$ during the course of the spread of West Asian domesticates into peninsular India [40] and (b) the formation of Austroasiatic speaking populations through admixture between the eastward migrating branch of out of Africa populations that arrived in South Asia $~ 3000$ BCE and ancient indigenous Indian groups (AASI-related) [17, 18].

In brief, we found very little similarity between Kumhars and other ethnic groups from the same geographic regions in Uttar Pradesh and its adjoining state of Uttarakhand, in north India. The Kumhars appeared to have 
an overwhelming ASI ancestral component; 24 out of 27 Kumhars appeared to be identical (>98\%) to a small population known as the Kurchas from the Wayanad district in the south Indian state of Kerala, which is approximately $2500 \mathrm{~km}$ south of the region from where the Kumhar samples were obtained. Similar to the Kumhars little is known regarding the Kurcha population and to the best of our knowledge there is no existing literature that describes any anthropological or historical connection between Kurchas with either the Kumhars or the Kulalas.

Here, we propose that the significant genomic similarity between two apparently distinct modern day Indian populations that correspond to well separated geographical areas separated by $\sim 2500 \mathrm{~km}$ with no known overlapping history or links likely alludes to their common origin during or after the decline of IVC; subsequently the two populations likely migrated towards opposite ends of the Indian subcontinent but their genomic integrity was preserved owing to stringent enforcement of endogamy. Our findings illuminate the population history of two Indian groups, allowing a glimpse into one or few of numerous of human migrations that likely occurred across the Indian subcontinent and have shaped its varied and vibrant evolutionary past. Overall our findings help to reconstruct the genomic history of two Indian populations, the Kumhars and Kurchas, which despite significant geographic isolation have remained almost identical, genetically, shining light on how largescale population movements that spanned across the Indian subcontinent over extensive periods of time together with imposition of sociocultural hierarchies have contributed to its diverse evolutionary heritage.

Previous reports have indicated that the IBD scores for Kumhars are significantly higher than that of the Ashkenazi Jews and the Finns, consistent with social practices of consanguinity among them [23]. This is medically relevant as it predicts a high propensity for genetic disorders and consistent with this diseases such as acute intermittent porphyria are reported at higher frequencies in the Kumhar population [41].

\section{Limitations of the study}

As mentioned earlier, Kumhars are distributed throughout North India and Pakistan. However, since we did not genotype/sequence any Kumhar sample and our study was completely based on previously published SNP genotype data, we were limited in terms of sample size and distribution. The same is applicable for the Kurchas. We could only employ four Kurcha samples in this study due to unavailability of Kurchas in the published datasets. It can be speculated that sampling across various parts of India can significantly improve the robustness of the study.

\section{Conclusions}

Overall, our results reflect the high genomic similarity of Kumhars with various south Indian groups, including the so far little known Kurchas, offering some insight into the latter's genomic history and likely predisposition to genetic disorders. It further underscores the importance of uncovering founder events among Indian populations and prioritizing them for studies dissecting genetic diseases and their underlying etiology.

\section{Methods}

\section{Data sets}

To generate the Modern South Asian only dataset we merged two previously published datasets $[16,23]$ using 'mergeit' function implemented in EIG v7.2 [42]. This dataset comprised of 2040 modern South Asian SNP genotype data, including 27 Kumhar samples, and corresponding to total 265 Indian ethnic groups, and assessing 91,781 single nucleotide polymorphisms (SNPs). The Modern South Asian only dataset was then merged with two ancient DNA datasets comprising of 294 and 362 ancient individuals, respectively $(N=2696)$ [17, 40]. Finally, this dataset was merged with 1879 modern samples [43] across the world to generate the Ancient-Modern dataset comprising of 4575 individuals, and assessing 91,768 SNPs. File conversions and manipulations were performed using EIG v7.2 [42] and PLINK v1.9 [44] (https://www.cog-genomics.org/plink2/).

\section{Genome-wide SNP data analyses}

Modern South Asian only dataset $(N=2040)$ was used for all genome-wide SNP analyses to describe fine-scale population structure recapitulating the population history of Kumhars.

We calculated mean and weighted pairwise $F_{\mathrm{ST}}$ between Kumhars and 63 selected populations across India using the Weir and Cockerham approach [27] implemented in PLINK v1.9 [44]. PLINK estimated the fixation indices separately for all 91,781 SNPs under evaluation using -fst command alongside -family flag that enables it to group the individuals according to their family id (FID). The 63 populations comprised of all 35 available populations from Uttarakhand and Uttar Pradesh, all 10 populations from Kerala, and 18 populations from elsewhere in India.

The fine population structure of the modern South Asians was delineated using Principal Component Analysis (PCA) implemented in PLINK v1.9 [44] using -pca command. The two most informative PCs are discussed and plotted in R v3.5.1.

The genomic ancestry of all 2040 individuals was estimated using the model-based clustering algorithm ADMIXTURE v1.3 [28]. The optimum number of ancestral components $(K)$ was determined by minimizing the 
cross-validation error (CVE) using a -cv flag to the admixture command line. The lowest CVE was estimated for $K=11$ (Additional file 1 Fig. S1).

We constructed a maximum likelihood (ML) tree for 84 selected populations comprising of all 35 populations from Uttar Pradesh and Uttarakhand, all 10 populations from Kerala, and 39 populations across the rest of India by using TreeMix v.1.12 [29] in order to place Kumhars to a global context. Onge were used to root the ML tree.

We applied ALDER v.1.02 [30] to compute a weighted linkage disequilibrium (LD) analysis to infer the likely date of admixture, based on the exponential LD decay. We aimed to investigate the approximate time of admixture between Kumhars and other South Asians considering a generation time of 28 years. Kumhars were included as the 'admixpop' (admixed population) and the remaining South Asian populations present in the Modern South Asian only dataset were used as the 'refpops' (reference populations).

\section{Biogeographical mapping of Kumhar samples}

Biogeographical analysis was performed using the Geographical Population Structure (GPS) algorithm, which has been successfully used to reconstruct history of several populations worldwide [45-53]. GPS correlates the admixture patterns of individuals of unknown origins using the admixture fractions (GEN file) and geographical locations or coordinates (GEO file) of reference individuals with known geographical origin. GPS converts the genetic distances between the query and the most proximal reference populations into geographic distances. Comparing the admixture proportions of the query with the reference populations, GPS extrapolates the genomic similarity of the former and infers its geographic origins using the known biogeographical information of the reference. Our test dataset comprised of the admixture fractions of Kumhars. We curated the reference dataset using the rest of south Asians present in the Modern South Asian only dataset except Siddis and Ashkenazi Jews.

\section{Determination of ancestry proportions in Kumhars in a global context}

We used qpAdm [32] implemented in AdmixTools v5.1 [33] to estimate ancestry proportions in the South Asians originating from a mixture of 'reference' populations by utilizing shared genetic drift with a set of 'outgroup' populations. The Ancient-Modern dataset comprising of 4575 ancient and modern-day samples from across the world was employed for this analysis. In accordance with existing literature [17], three ancient samples namely Shahr-iSoktha_MLBA2, Shahr-i-Soktha_MLBA3 and Gonur2_BA were referred to as 'Indus_Periphery' in $q p A d m$ analysis. All South Asians were modelled as a combination of three source populations namely Andaman Islanders (Onge), Steppe-related (Steppe-MLBA) and Iran-Turan-related (Indus_Periphery) as Left (Test, Onge, Steppe-MLBA, Indus_Periphery) as already described [17]. We used a mixture of eight ancient and modern-day populations comprising of Mabuti.DG, SHG, EHG, Ganj_Dareh_N, Anatolia_N, West_Siberia_N, Han and Karitiana our 'Right' outgroup populations (O8).

\section{The direction of gene flow}

To investigate whether Kumhar is genetically closer to Kurcha than to any other South Asian group used in this study, we employed qpDstat function implemented in AdmixTools v5.1 [33] in order to acquire information about the gene flow among South Asian population(s) in respect to Kumhar and Kurcha. The D-statistic was modelled as:

Pop1 (Kumhar) Pop2 (Modern South Asian populations): Pop3 (Kurcha) Pop4 (Onge).

Onge was used as an outgroup since it has been disconnected from the mainland populations long while ago. Here, while positive Z-scores will indicate gene flow between Kumhar and Kurcha, negative scores will indicate gene flow between other South Asian population(s) and Kurcha.

\section{Supplementary information}

Supplementary information accompanies this paper at https://doi.org/10. 1186/s12863-020-00919-2.

Additional file 1. Supplementary Material

\section{Abbreviations}

IVC: Indus Valley Civilization; AASI: Ancient Ancestral South Indian; ANI: Ancestral North Indian; ASI: Ancestral South Indian; ATB: Ancestral Tibeto-Burman; AAA: Ancestral Austro-Asiatic; Steppe MLBA: Steppe MiddleLate Bronze Age; IBD: Identical by Descent

\section{Acknowledgements}

The authors are grateful to the SBB-2019 Committee for support of this work.

About this supplement

This article has been published as part of BMC Medical Genetics Volume 21 Supplement 1, 2020: Selected Topics in "Systems Biology and Bioinformatics" - 2019: medical genetics. The full contents of the supplement are available online at https://bmcmedgenet.biomedcentral.com/articles/supplements/ volume-21-supplement-1.

Authors' contributions

$\mathrm{RD}$ conceived the idea of the work. RD did the analysis with inputs from PU. $\mathrm{VI}$ and AA helped in the computational analyses. PU wrote the manuscript. All the authors finalized the manuscript. The author(s) read and approved the final manuscript.

\section{Funding}

The publication cost was covered by Russian Ministry of Education and Science, Project No. 28.12487.2018/12.1. "Investigation, analysis and complex independent expertise of projects of the National technological initiatives, including the accompanying of projects of «road map» «NeuroNet»". The funding body had no role in design of the study, analysis, data interpretation and writing the manuscript. 


\section{Availability of data and materials}

While most data are publicly available through the lab database of Dr. David Reich, Harvard University (https://reich.hms.harvard.edu/datasets) in Eigenstrat format, some were obtained from Dr. Reich's lab through personal communication. The authors do not have the mandate to redistribute these data. Kindly contact Dr. Ranajit Das (das.ranajit@gmail.com) for details regarding the data availability.

\section{Ethics approval and consent to participate}

The SNP genotype data for the current study was obtained from previously published datasets. The original authors collected the samples under the supervision of ethical review boards in India with informed consent obtained from all subjects and sample collection was performed in accordance with the ethical standards of the responsible committees on human experimentation.

\section{Consent for publication}

Not applicable.

\section{Competing interests}

The authors declare that they have no competing interests.

\section{Author details}

'Yenepoya Research Centre (YRC), Yenepoya (Deemed to be University), Mangalore, Karnataka, India. ${ }^{2}$ Humanitarian Institute, Novosibirsk State University, 630090 Novosibirsk, Russia. Institute of Cytology and Genetics SB RAS, Novosibirsk, Russia. ${ }^{4}$ The Digital Health Institute, I.M. Sechenov First Moscow State Medical University (Sechenov University), Moscow, Russia. ${ }^{5}$ Engelhardt Institute of Molecular Biology RAS, Moscow, Russia. ${ }^{6}$ Department of Medical Genetics, Kasturba Medical College, Manipal Academy of Higher Education, Manipal, Karnataka, India.

\section{Published: 22 October 2020}

\section{References}

1. Gangal K, Sarson GR, Shukurov A. The near-eastern roots of the Neolithic in South Asia. PLoS One. 2014:9(5):e95714.

2. Kivisild T. In: Papiha SS, Deka R, Chakraborty R, editors. Genomic Diversity: Applications in Human Population Genetics. New York: Kluwer; 2001. p. 135-52.

3. Metspalu M, Kivisild T, Metspalu E, Parik J, Hudjashov G, Kaldma K, Serk P, Karmin M, Behar DM, Gilbert MT, et al. Most of the extant mtDNA boundaries in south and Southwest Asia were likely shaped during the initial settlement of Eurasia by anatomically modern humans. BMC Genet. 2004;5:26.

4. Misra VN. Prehistoric human colonization of India. J Biosci. 2001;26(4 Suppl): 491-531.

5. Singh S, Singh A, Rajkumar R, Sampath Kumar K, Kadarkarai Samy S, Nizamuddin S, Singh A, Ahmed Sheikh S, Peddada V, Khanna V, et al. Dissecting the influence of Neolithic demic diffusion on Indian Ychromosome pool through J2-M172 haplogroup. Sci Rep. 2016;6:19157.

6. Thangaraj K, Chaubey G, Singh VK, Vanniarajan A, Thanseem I, Reddy AG, Singh L. In situ origin of deep rooting lineages of mitochondrial Macrohaplogroup ' $M$ ' in India. BMC Genomics. 2006;7:151.

7. Mellars P. Going east: new genetic and archaeological perspectives on the modern human colonization of Eurasia. Science. 2006:313(5788):796-800.

8. Macaulay V, Hill C, Achilli A, Rengo C, Clarke D, Meehan W, Blackburn J, Semino O, Scozzari R, Cruciani F, et al. Single, rapid coastal settlement of Asia revealed by analysis of complete mitochondrial genomes. Science. 2005:308(5724):1034-6

9. Quintana-Murci L, Semino O, Bandelt HJ, Passarino G, McElreavey K, Santachiara-Benerecetti AS. Genetic evidence of an early exit of Homo sapiens sapiens from Africa through eastern Africa. Nat Genet. 1999;23(4): 437-41.

10. Sengupta S, Zhivotovsky LA, King R, Mehdi SQ, Edmonds CA, Chow CE, Lin AA, Mitra M, Sil SK, Ramesh A, et al. Polarity and temporality of highresolution $y$-chromosome distributions in India identify both indigenous and exogenous expansions and reveal minor genetic influence of central Asian pastoralists. Am J Hum Genet. 2006;78(2):202-21.

11. Basu A, Mukherjee N, Roy S, Sengupta S, Banerjee S, Chakraborty M, Dey B, Roy M, Roy B, Bhattacharyya NP, et al. Ethnic India: a genomic view, with special reference to peopling and structure. Genome Res. 2003;13(10):2277-90.
12. Bamshad M, Kivisild T, Watkins WS, Dixon ME, Ricker CE, Rao BB, Naidu JM Prasad BV, Reddy PG, Rasanayagam A, et al. Genetic evidence on the origins of Indian caste populations. Genome Res. 2001;11(6):994-1004.

13. Consortium HP-AS, Abdulla MA, Ahmed I, Assawamakin A, Bhak J, Brahmachari SK, Calacal GC, Chaurasia A, Chen CH, Chen J, et al. Mapping human genetic diversity in Asia. Science. 2009:326(5959):1541-5.

14. Basu A, Sarkar-Roy N, Majumder PP. Genomic reconstruction of the history of extant populations of India reveals five distinct ancestral components and a complex structure. Proc Natl Acad Sci U S A. 2016;113(6):1594-9.

15. Reich $D$, Thangaraj K, Patterson N, Price AL, Singh L. Reconstructing Indian population history. Nature. 2009:461(7263):489-94.

16. Moorjani $P$, Thangaraj $K$, Patterson N, Lipson M, Loh PR, Govindaraj P, Berger B, Reich D, Singh L. Genetic evidence for recent population mixture in India. Am J Hum Genet. 2013;93(3):422-38.

17. Narasimhan VM, Patterson NJ, Moorjani P, Lazaridis I, Mark L, Mallick S, Rohland N, Bernardos R, Kim AM, Nakatsuka N, et al. The Genomic Formation of South and Central Asia. bioRxiv. 2018:292581.

18. Mallick S, Li H, Lipson M, Mathieson I, Gymrek M, Racimo F, Zhao M, Chennagiri N, Nordenfelt S, Tandon A, et al. The Simons genome diversity project: 300 genomes from 142 diverse populations. Nature. 2016;538(7624): 201-6.

19. Kathayat G, Cheng H, Sinha A, Yi L, Li X, Zhang H, Li H, Ning Y, Edwards RL. The Indian monsoon variability and civilization changes in the Indian subcontinent. Sci Adv. 2017;3(12):e1701296.

20. Underhill PA, Poznik GD, Rootsi S, Jarve M, Lin AA, Wang J, Passarelli B, Kanbar J, Myres NM, King RJ, et al. The phylogenetic and geographic structure of $\mathrm{Y}$ chromosome haplogroup R1a. Eur J Hum Genet. 2015;23(1):124-31.

21. Mathieson I, Alpaslan-Roodenberg S, Posth C, Szecsenyi-Nagy A, Rohland N, Mallick S, Olalde I, Broomandkhoshbacht N, Candilio F, Cheronet $\mathrm{O}$, et al. The genomic history of southeastern Europe. Nature. 2018;555(7695):197-203.

22. Silva M, Oliveira M, Vieira D, Brandao A, Rito T, Pereira JB, Fraser RM, Hudson B, Gandini F, Edwards C, et al. A genetic chronology for the Indian subcontinent points to heavily sex-biased dispersals. BMC Evol Biol. 2017;17(1):88.

23. Nakatsuka N, Moorjani P, Rai N, Sarkar B, Tandon A, Patterson N, Bhavani GS, Girisha KM, Mustak MS, Srinivasan S, et al. The promise of discovering population-specific disease-associated genes in South Asia. Nat Genet. 2017; 49(9):1403-7.

24. Mandal SK: Kumhar/Kumbhar. In: People of India: Rajasthan. Edited by Singh SK: Popular Prakashan; 1998: 565-566

25. Saraswati B. Pottery-making cultures and Indian civilization: Abhinav publications; 1979

26. Das R, Upadhyai P. An ancestry informative marker set which recapitulates the known fine structure of populations in South Asia. Genome Biol Evol. 2018:10(9):2408-16.

27. Weir BS, Cockerham CC. Estimating F-statistics for the analysis of population structure. Evolution. 1984:38(6):1358-70

28. Alexander DH, Novembre J, Lange K. Fast model-based estimation of ancestry in unrelated individuals. Genome Res. 2009:19(9):1655-64.

29. Pickrell JK, Pritchard JK. Inference of population splits and mixtures from genome-wide allele frequency data. PLoS Genet. 2012:8(11):e1002967.

30. Loh PR, Lipson M, Patterson N, Moorjani P, Pickrell JK, Reich D, Berger B. Inferring admixture histories of human populations using linkage disequilibrium. Genetics. 2013;193(4):1233-54.

31. Brooke JL. Climate change and the course of global history: a rough journey: Cambridge University press; 2014.

32. Haak W, Lazaridis I, Patterson N, Rohland N, Mallick S, Llamas B, Brandt G, Nordenfelt S, Harney E, Stewardson K, et al. Massive migration from the steppe was a source for indo-European languages in Europe. Nature. 2015; 522(7555):207-11.

33. Patterson N, Moorjani P, Luo Y, Mallick S, Rohland N, Zhan Y, Genschoreck T, Webster T, Reich D. Ancient admixture in human history. Genetics. 2012 192(3):1065-93.

34. Majumder PP. Ethnic populations of India as seen from an evolutionary perspective. J Biosci. 2001;26(4 Suppl):533-45.

35. Majumder PP. The human genetic history of South Asia. Curr Biol. 2010; 20(4):R184-7.

36. Sun C, Kong QP, Palanichamy MG, Agrawal S, Bandelt HJ, Yao YG, Khan F, Zhu CL, Chaudhuri TK, Zhang YP. The dazzling array of basal branches in the mtDNA macrohaplogroup M from India as inferred from complete genomes. Mol Biol Evol. 2006;23(3):683-90. 
37. Cahille MA. Paradise rediscovered: the roots of civilisation: interactive publications; 2012.

38. Langer WL. An encyclopedia of world history. Boston: Houghton Mifflin Company; 1972.

39. Southworth F. Linguistic archaeology of South Asia Routledge; 2005

40. Kenoyer JM. Ancient cities of the Indus Valley civilization. Karachi: Oxford University Press; 1998.

41. Sachdev R, Haldiya KR, Dixit AK. Acute intermittent Porphyria in a Kumhar community of Western Rajasthan. J Assoc Physicians India. 2005;53:101-4.

42. Price AL, Patterson NJ, Plenge RM, Weinblatt ME, Shadick NA, Reich D.

Principal components analysis corrects for stratification in genome-wide association studies. Nat Genet. 2006;38(8):904-9.

43. Lazaridis I, Nadel D, Rollefson G, Merrett DC, Rohland N, Mallick S, Fernandes D, Novak M, Gamarra B, Sirak K, et al. Genomic insights into the origin of farming in the ancient near east. Nature. 2016;536(7617):419-24.

44. Purcell S, Neale B, Todd-Brown K, Thomas L, Ferreira MA, Bender D, Maller J, Sklar P, de Bakker PI, Daly MJ, et al. PLINK: a tool set for whole-genome association and population-based linkage analyses. Am J Hum Genet. 2007; 81(3):559-75.

45. Elhaik E, Tatarinova T, Chebotarev D, Piras IS, Maria Calo C, De Montis A, Atzori M, Marini M, Tofanelli S, Francalacci P, et al. Geographic population structure analysis of worldwide human populations infers their biogeographical origins. Nat Commun. 2014;5:3513.

46. Marshall S, Das R, Pirooznia M, Elhaik E. Reconstructing Druze population history. Sci Rep. 2016;6:35837.

47. Das R, Wexler P, Pirooznia M, Elhaik E. The origins of Ashkenaz, Ashkenazic Jews, and Yiddish. Front Genet. 2017;8:87.

48. Das R, Upadhyai P. Application of geographic population structure (GPS) algorithm for biogeographical analyses of populations with complex ancestries: a case study of south Asians from 1000 genomes project. BMC Genet. 2017;18(Suppl 1):109.

49. Das R, Upadhyai P. Adaptation of the Geographic Population Structure (GPS) algorithm for biogeographical analyses of wild and captive Gorillas. BMC Bioinformatics (In Press). 2019;20:35.

50. Das R, Wexler P, Pirooznia M, Elhaik E. Localizing Ashkenazic Jews to primeval villages in the ancient Iranian lands of Ashkenaz. Genome Biol Evol. 2016;8(4):1132-49.

51. Aberg KA, Chan RF, Shabalin AA, Zhao M, Turecki G, Staunstrup NH, Starnawska A, Mors O, Xie LY, van den Oord EJ. A MBD-seq protocol for large-scale methylome-wide studies with (very) low amounts of DNA. Epigenetics. 2017;12(9):743-50.

52. Flegontov P, Changmai P, Zidkova A, Logacheva MD, Altinisik NE, Flegontova O, Gelfand MS, Gerasimov ES, Khrameeva EE, Konovalova OP, et al. Genomic study of the Ket: a Paleo-Eskimo-related ethnic group with significant ancient north Eurasian ancestry. Sci Rep. 2016;6:20768.

53. Triska P, Chekanov N, Stepanov V, Khusnutdinova EK, Kumar GPA Akhmetova V, Babalyan K, Boulygina E, Kharkov V, Gubina M, et al. Between Lake Baikal and the Baltic Sea: genomic history of the gateway to Europe. BMC Genet. 2017;18(Suppl 1):110.

\section{Publisher's Note}

Springer Nature remains neutral with regard to jurisdictional claims in published maps and institutional affiliations.

Ready to submit your research? Choose BMC and benefit from:

- fast, convenient online submission

- thorough peer review by experienced researchers in your field

- rapid publication on acceptance

- support for research data, including large and complex data types

- gold Open Access which fosters wider collaboration and increased citations

- maximum visibility for your research: over $100 \mathrm{M}$ website views per year

At $\mathrm{BMC}$, research is always in progress.

Learn more biomedcentral.com/submissions 